\title{
EXPLORE USER BEHAVIOUR IN SEMI-AUTONOMOUS DRIVING
}

\author{
Shi, Yuan; Maskani, Jeyhoon; Caruso, Giandomenico; Bordegoni, Monica \\ Politecnico di Milano
}

\begin{abstract}
The control shifting between a human driver and a semi-autonomous vehicle is one of the most critical scenarios in the road-map of autonomous vehicle development. This paper proposes a methodology to study driver's behaviour in semi-autonomous driving with physiological-sensors-integrated driving simulators. A virtual scenario simulating take-over tasks has been implemented. The behavioural profile of the driver has been defined analysing key metrics collected by the simulator namely lateral position, steering wheel angle, throttle time, brake time, speed, and the take-over time. In addition, heart rate and skin conductance changes have been considered as physiological indicators to assess cognitive workload and reactivity. The methodology has been applied in an experimental study which results are crucial for taking insights on users' behaviour. Results show that individual different driving styles and performance are able to be distinguished by calculating and elaborating the data collected by the system. This research provides potential directions for establishing a method to characterize a driver's behaviour in a semi-autonomous vehicle.
\end{abstract}

Keywords: Virtual reality, Simulation, Evaluation, Semi-autonomous vehicle, User behaviour

\author{
Contact: \\ Shi, Yuan \\ Politecnico di Milano \\ Department of Mechanical Engineering \\ Italy \\ yuan.shi@polimi.it
}

Cite this article: Shi, Y., Maskani, J., Caruso, G., Bordegoni, M. (2019) 'Explore User Behaviour in Semi-autonomous Driving', in Proceedings of the 22nd International Conference on Engineering Design (ICED19), Delft, The Netherlands, 5-8 August 2019. DOI:10.1017/dsi.2019.394 


\section{INTRODUCTION}

Driving simulator has been used in various of researches to improve the driving safety and user experience. As a tool to study the interaction between driver and vehicle, it permits a controllable, reproducible and cost-effective user behaviour experimental environment (Drosdol and Panik, 1985). Attributed to the increasing automation level of vehicle control system, the conventional way of interaction between driver and vehicle is changing (Merat et al., 2014; Banks and Stanton, 2017). Among all the automation levels, the most critical scenario appears the one related to semi-autonomous vehicles (L2-L3 of SAE international). These vehicles allow driver to have their hands and feet free but monitoring the movement of vehicle, and requires the driver get back control in a few seconds when the system reaches the limits of its Operational Design Domain (ODD). The ODD may include geographic, roadway, environmental, traffic, speed, and/or temporal limitations of automated driving availability and the role of the user shifts from the passenger to the driver when the Dynamic Driving Task (DDT) changes from Automated Driving System (ADS) to the manual one (SAE J3016, 2016). It becomes crucial to understand if the user is prepared for take-over in a few seconds both from cognitive and performing level.

The cognitive workload of the driver is generally be assessed in 4 methods: physiological measurement (the physiological measurement may include electrodermal, cardiovascular and respiratory activity; Heikoop et al., 2018 ), secondary task engagement (Naujoks et al., 2016; Reimer and Mehler, 2011), self-reported test (the self-reported test may include self-reported overall workload, e.g. the NASA Task Load Index(TLX), and standardized change scores of self-reported stress(DSSQ); Heikoop et al., 2018), and performance measurement (the performance metrics may include lane position, steering wheel angle, brake time, following distance, driving speed, and recovery time; Eriksson and Stanton, 2017; Strayer and Drews, 2004). A synthetic matrix of these methods may obtain an insight of the driver's state in a relatively wider perspective.

The aim of this study is to define a method to explore user behaviour in the context of semiautonomous driving with multiple measurements. By using driving simulator and virtual reality in laboratory environment, user's behavioural data can be collected continuously along all the entire 'hand-over' to 'take-over' process in the semi-autonomous driving, for instance: driver performance data i.e. standard deviation of lateral position (SDLP), standard deviation of steering wheel angle (SDSW), and mean amplitude of steering wheel (SWM), which are automatically recorded and can be extracted from the simulator; and user's physiological data i.e. electrodermal and cardiovascular activity, which can be recorded continuously with the simulate scenario and interpreted to describe user's relative cognitive workload.

\section{BACKGROUND}

According to SAE J3063, for ADS of Level 2 (L2) namely Partial Driving Automation and Level 3 (L3) namely Conditional Driving Automation, the ADS is in charge of lateral and longitudinal vehicle motion control subtasks of the (DDT), while L2 possesses only limited the Object Event Detection and Response (OEDR) subtask but L3 the system completes the DDT task with the OEDR task. In both cases, the human user performs either a portion of the DDT or the DDT fallbacks temporarily.

There are already commercially available vehicles on the road with a semi-autonomous system according to the previous taxonomy of ADS, for instance 'Autopilot' of Tesla (Tesla Motors, 2016), and Mercedes 'Distronic Plus with Steering Assist' (Mercedes-Benz, 2013). In the Tesla Model S, the ADS is based on the sensors to detect the circumstance, so the ADS becomes available for the user when the lane mark is recognizable and driving speed is beyond $30 \mathrm{~km} / \mathrm{h}$. The user may initiate a handover to the ADS at any time while the access of ADS is open. And when the lane mark is inrecognizable by the sensors, or in any other case that the ADS reaches its limit, the user need to resume the control of the car promptly following the alarm given by the ADS. In the semi-autonomous driving, the Automated Driving System (ADS) is available conditionally, in which case there is the possibility for the user to hand-over the control to ADS and vice versa. The initiation of the transfer can be of two types: one is 'User-Initiated' and the other is 'System-Initiated' (SAE J3114, 2016). For the human user-initiated transfer, the user indicates to the ADS that a transfer of control is desired, either human-to-system or system-to-human. The user-initiated transfer can be categorized into intended, unintended (the unintended user-initiated transfer that all or a portion of the DDT is transferred by the user unknowingly, usually being defined as an operational error; Banks et al., 2018), non-permitted (non-permitted transfer refers that the ADS prevents the transfer of control when the 
situation is deemed unsafe by the system) and deferred (the deferred transfer of control occurs after a designated amount of time when the request is delivered, for the safety or driver readiness) . The systeminitiated transfer can be classified into four conditions (from the user's point of view): scheduled (the scheduled transfer of the control is an expected one based on the operational design domain and the environmental conditions), imminent (the imminent transfer of control is an unexpected transfer due to the operational design domain and the environment), nuisance (the nuisance transfer of control is initiated by the system when it is not wanted for the situation), and request to intervene (the Request to intervene is a notification by the ADS to indicate the driver perform the DDT fallback promptly).

In this paper, the intended user-initiated transfer and request to intervene are introduced in the design of the scenario, in order to provide a realistic driving scenario in the simulator.

As previously introduced, the DDT in semi-autonomous driving performs the lateral and longitudinal movement, and a portion or all of the OEDR subtasks. Putting into the hierarchical structure of the vehicle control loops defined in SAE J3016(2016) (e.g. Figure 1 Multi-level control in driving; Merat et al., 2018), the innermost loop is performed by the ADS, and the partial or all of the middle loop is performed by the ADS. The role of the user is to complete the loops with or without the ADS in which manner the DDT can be completed.

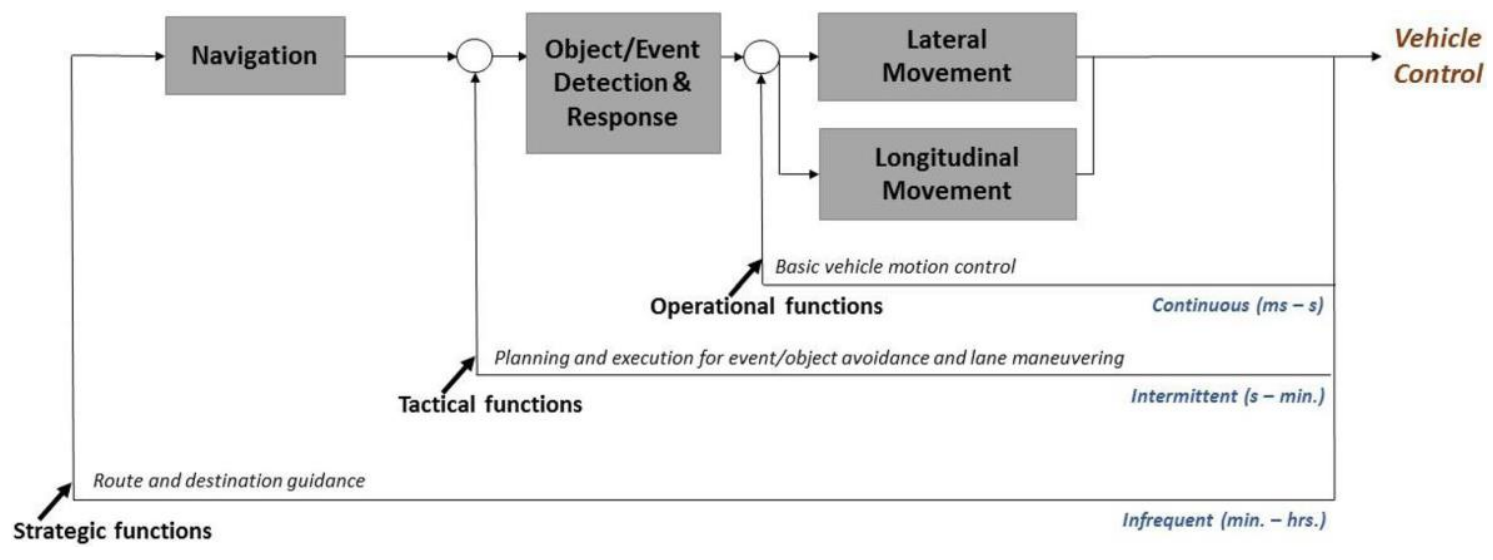

Figure 1. Multi-level control in driving (Merat et al., 2018)

"Out of the loop" is a term frequently used in recent literature when considering the effect of ADS on driver behaviour and performance from a perspective of human factor (Shen and Neyens, 2017). It has been used to define a state of driver when the vehicle is physically out of control from the driver but under the supervision of the driver (e.g., Saffarian et al., 2012; Strand et al., 2014), or a state that the driver is mentally not active to monitor the system and driving circumstances, which means that the driver is beyond the inner two loops (e.g. Casner et al., 2016; Louw and Merat 2017). A more precise definition of the states of the driver has been proposed by Merat et al. (2018):

- In the loop: In physical control of the vehicle and monitoring the driving situation

- On the loop: Not in physical control of the vehicle, but monitoring the driving situation.

- Out of the loop: Not in physical control of the vehicle, and not monitoring the driving situation, OR in physical control of the vehicle but not monitoring the driving situation.

When the ADS performs the DDT while the user is out of the loop in a semi-autonomous vehicle, the user is potentially not able to react properly to a take-over request from the system.

\section{PROPOSED METHOD}

The method proposed in this study includes the implementation of a specific virtual scenario, where the behaviour of a semi-autonomous vehicle is simulated and the use of a driving simulator including sensors allows gathering physiological data form the driver during the test.

Numerous studies of human factors and driver behaviour have been conducted using driving simulators (de Winter et al., 2012; Shen and Neyens, 2017) since the experimental environment can be carefully controlled and the experiments can be conducted in a safer way respect to the real context. In the field of driver's behavioural analysis, including behavioural data and physiological data, results gathered with driving simulators can be compared with on-road experimental test, as demonstrated by Ruscio et al. (2017). 
As introduced above, semi-autonomous driving scenario is composed by two transitions of the control of system (Figure 2): the first one is manual to Automated Driving (AD) control, while the second one is $\mathrm{AD}$ to manual control. The manual to $\mathrm{AD}$ control transition is an intended user-initiated transfer. The AD to manual control transition is a request to intervene initiated by the ADS. The entire scenario consists 9 state phases in the time series. From phase 0 to phase 2 and in phase 8 the Automation is inactive, while from phase 4 to phase 6 the Automation is in active mode. For the safety purpose, the $\mathrm{ADS}$ is expected to engage the control of the vehicle when the automation is available as soon as possible, in order to keep the minimum timeslot of Phase 2; and users are expected to resume the manual control from the ADS in Phase 6 in an as short as possible time.

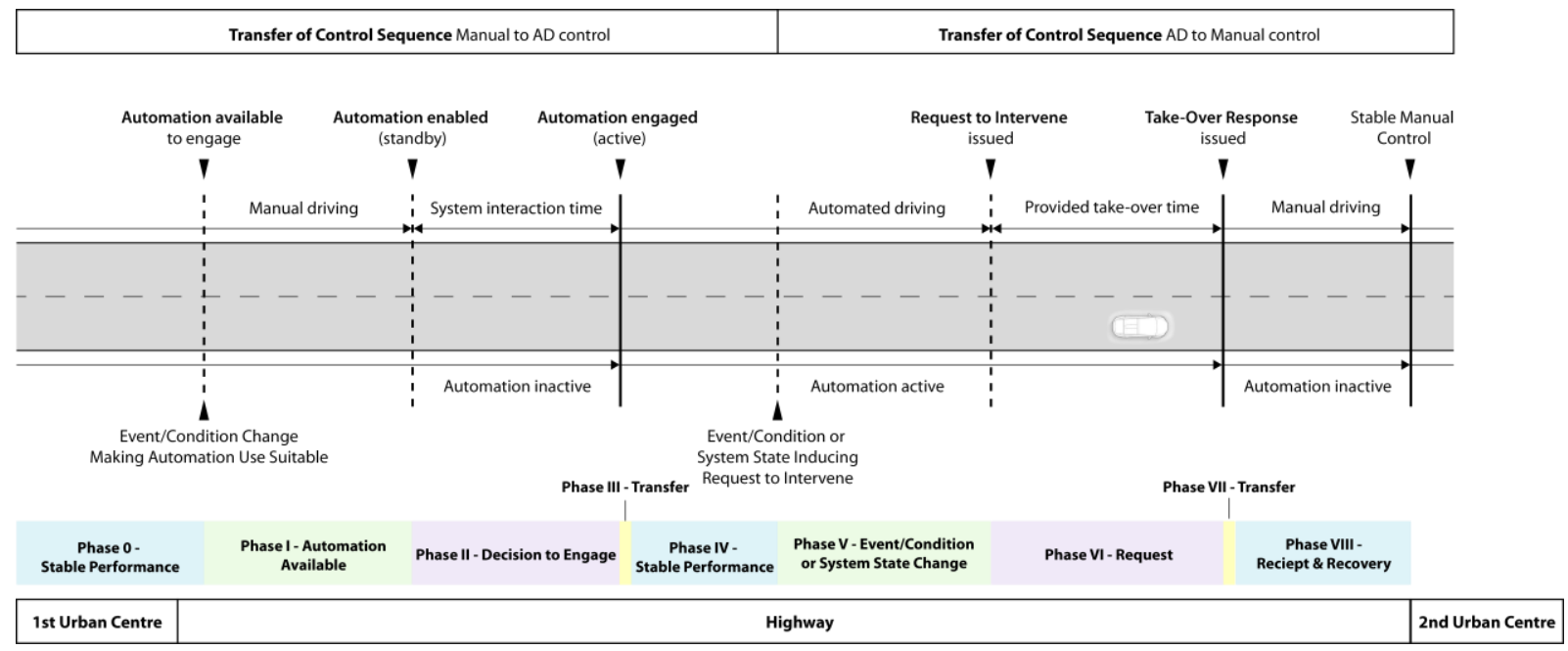

Figure 2. Schematic task sequence of semi-autonomous driving scenario, adapted from Fig. 5 and Fig. 7 in SAE J3114 (2016)

The proposed driving scenario has been developed by adapting the schematic task sequence to a possible real context. As a result, the scenario includes two urban centres, where the driver manually conducts the vehicle, connected by a highway, where the ADS is available. The two transitions of the control of system occur in the highway section as shown in the bottom of Figure 2.
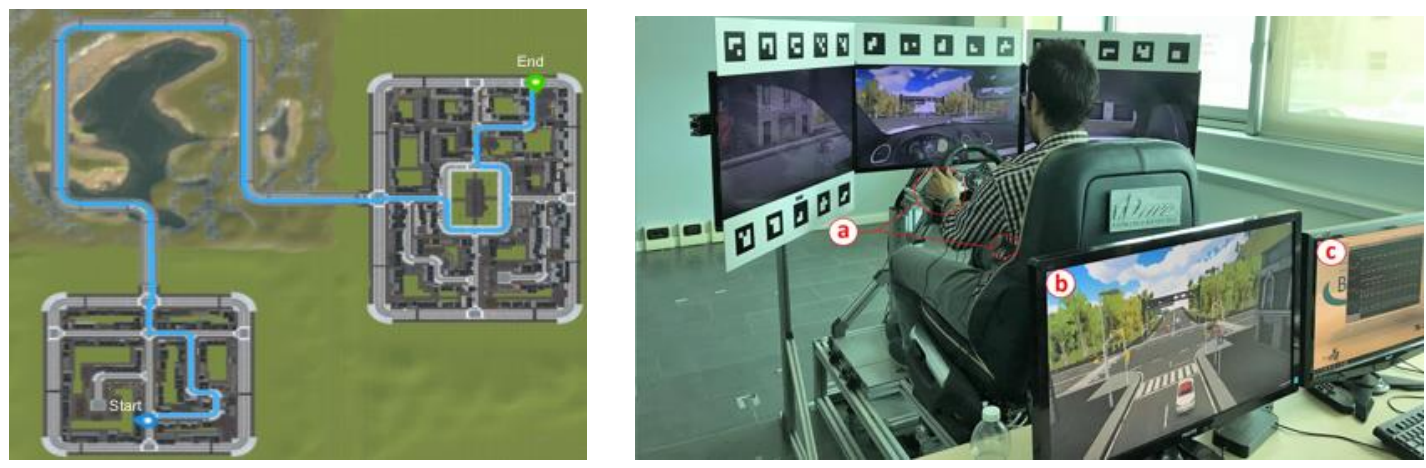

Figure 3. Left: Bird view of the route in the scenario in Unity 3D; Right: Driving Simulator Setup. a: Body-worn sensors. b: Used for monitoring the driver, and as a control panel for the simulator. c: system for recording physiological data

When the car drives into the highway and maintains a stable behaviour, the system is ready to enable the $\mathrm{AD}$, and before the car enters in the second urban centre, the system requires the user to resume manual control of the car. A map of the scenario including the path followed during the test is reported in (Figure 3, Left). The virtual driving scenario was built according to the task sequence by using (Unity 3D) which is a game engine, widely used in various studies for creating virtual experimental environment for the automotive sector (Taheri et al., 2017).

The driving simulator, used during the test, consists of physical structure including a set of commercial vehicle control such as the steering wheel with force feedback, the gear shift lever with automatic transmission, and the brake and accelerator pedals (Logitech G920; Figure 3, Right). Three 32-inch screens providing 175 degrees field of view show the virtual driving scenario. 
The driving simulator is also equipped with the commercial physiological system Bio-graph Infinity (Thought Technology) to record skin conductance and EKG signals for identifying driver's state. Physiological measurements have been introduced in the design field as an effective objective method, for instance in (Yao et al., 2017) physiological data were collected and analysed as a complementary measure of user experience. They are currently used in driver behaviour studies for objectively measuring the user's reaction to the situation, stimuli and events. In order to have an insight on the different states of in the loop, on the loop and out of the loop of the user in diverse phases of the driving task, it is possible to measure the changes of mental elaboration effort that relates to the parasympathetic nervous system by considering the high frequency (between $0.15 \mathrm{~Hz}$ and $0.4 \mathrm{~Hz}$ ) component of heart rate variability, which has been elaborated from the EKG signal. At the meantime, the changes in the sympathetic nervous system that relates to cognitive arousal, as a strong predictor of attention and memory which may be utilized to detect the driver's awareness from the event and stimuli in driving situation, can be measured by changes in electrodermal activity of the skin (Brookhuis and Waard, 2009). Table 1 shows all the data collected during the test with the relative sampling rate.

Table 1. Data collected by driving simulator

\begin{tabular}{|c|c|c|c|}
\hline DATA & Unit & Description & $\begin{array}{l}\text { Sampling } \\
\text { rate }\end{array}$ \\
\hline World position $(\mathrm{X}, \mathrm{Y}, \mathrm{Z})$ & $\mathrm{m}$ & $\begin{array}{l}\text { Position of the car in the virtual driving } \\
\text { scenario }\end{array}$ & \multirow[t]{6}{*}{$10 \mathrm{~Hz}$} \\
\hline Speed & $\mathrm{Km} / \mathrm{h}$ & Speed of the car & \\
\hline Throttle & {$[0,1]$} & Axis of the Throttle pedal & \\
\hline Brake & {$[0,1]$} & Axis of the brake pedal & \\
\hline Self-driving mode & Boolean & Automated driving system active & \\
\hline Take-over request & Boolean & ADS initiates a transfer of control & \\
\hline Heart rate variability & $\mathrm{ms}$ & $\begin{array}{l}\text { Variation in heartbeats within a specific } \\
\text { timeframe }\end{array}$ & \multirow[t]{2}{*}{$8 \mathrm{~Hz}$} \\
\hline Skin conductance & $\mu \mathrm{S}$ & Electrodermal response of user & \\
\hline
\end{tabular}

\section{CASE STUDY}

The definition of user behaviour varies in different areas. In this case, it is defined as the way the user interacts with the semi-autonomous driving system, both physical behaviourally and cognitively. Moreover, the physical behavioural data can be recorded by the driving simulator and the physiological data can be recorded by the physiological measurement system. The realism of the semiautonomous driving scenario, along with the mandatory control shifting between user and system in different phases of the driving task, should elicit driver's behavioural changes in terms of physiological reactions and changing of the vehicle functional parameters. Human performance, also known as human reliability, is a term referring to the reliability of the human in a system, and it can be affected by many factors such as physical health, attitude, emotions, errors and cognitive biases (Gertman and Blackman, 1994). As a result, this case study aims at demonstrating the driver's driving style and performance in the semi-autonomous driving context can be evaluated by this driving simulator. The purpose of this study firstly is to verify the possibility of capturing human performance changes using the current driving simulation scenario; then furtherly explore the relationship between the user reaction to their performance parameters. Therefore, the research hypotheses are:

- H1: User performance can be assessed by behavioural data from this driving simulator;

- H2: There is a positive correlation between the take-over response time and the user performance.

Three healthy participants (Average age 27, Standard Deviation $(S D)=1$ ) who held a driver's license with a minimum of three years of driving experience volunteered for the experiment. All participants 
were graduate and post-graduate male students. None of them had ever used a driving simulator before the experiment. These three experiments are considered as a pilot-test to test the simulator setup for further improvement, and for future studies. Although the sample size is very small, the consistency of these three participants is high (considering age, experience, gender and background etc., which might affect the user performance). In the future, the variety of sample will be increased in order to get a more representative statistical result.

After the participants arrived in the laboratory, they were requested for the demographic data and signed the consent. Before starting, the participants were given a brief introduction into the functionality and controls of the driving simulator. Furthermore, each participant was asked to drive from the first urban centre to the second one following GPS indications. Before the experiment began, the participant was instructed to relax for three-minutes, sitting in the driver's seat while being equipped with physiological sensors for recording physiological activities under rest period as a resting baseline. Afterwards, they took ten minutes of free drive as an adaptation to the control of driving simulator and virtual driving environment. They were also given an adaptation scenario in order to practice the feature of transfer control (hand-over and take-over) with instruction. The adaptation scenario was introduced to familiarize the drive with car's user-interface (UI) and the self-driving mode, in order to minimize any novelty or learning effect that might be experienced by the participant during the test session.

Finally, the instruction about the driving task was repeated and each participant performed the test session. Physiological data alongside the simulation data were recorded for the driving sessions. Participants were also informed before that they could take-over the autonomous mode and drive manually whenever they uncomfortable driving autonomously. In the conclusion of the experiment, participants were asked to fill the Occupational Fatigue Inventory (SOFI-20) (Åhsberg et al., 1997) that consist of 20 Likert-type scale questions related to five dimensions of fatigue (i.e. lack of energy, physical discomfort, physical exertion, lack of motivation, and sleepiness), in order to give a subjective measurement on their driving experience.

Moreover, drivers were suggested to use self-driving mode on the highway whenever it available and whenever they comfortable with it. Visual indicator is used to inform driver about the availability of the self-driving mode, and visual alongside auditory signals were used for the take-over request as suggested in (Rau and Blanco, 2014).

\section{EXPERIMENTAL RESULTS AND DISCUSSION}

The data, which describe the driver's performance, are recorded and extracted from the driving simulator: time-distance comparison among participants (which describes the driver's performance in speed control), standard deviation of lateral position (SDLP), mean amplitude of lateral position (LPM), standard deviation of steering wheel angle (SDSW), and mean amplitude of steering wheel (SWM). Physiological measurements were integrated in the analysis, which provided a relatively non-invasive and continuous method to describe user's relative cognitive workload. High Frequency component of heart rate variability and Skin Conductance were measured in this experiment.

Figure 4 shows the distance each participant covered in the limited time of experiment, although the value of distance varies, it can be observed from the slop that driver number 2 demonstrated the best speed control aspect and then was Driver 1 and Driver 3, considering that they were requested to complete the task as soon as possible, obeying the safety requirement.

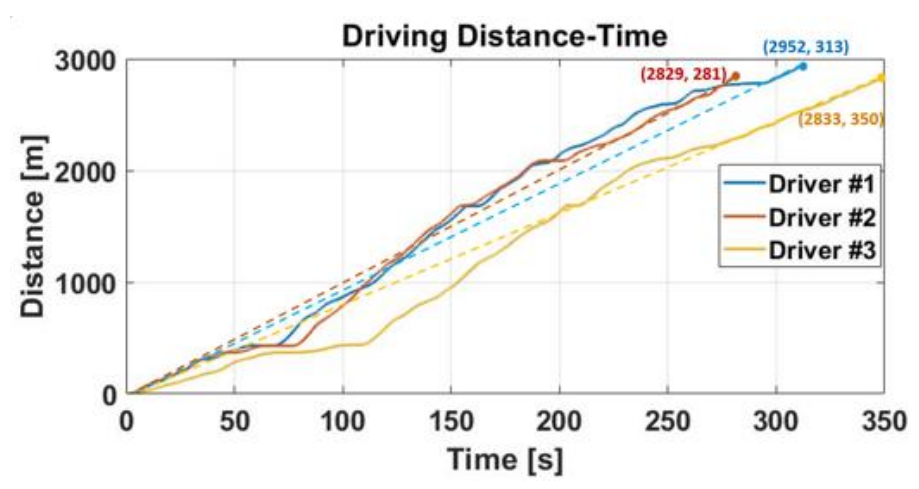

Figure 4. Time-distance comparison among participants 
SDLP is considered as the index for lane-keeping performance (lower the number, better the performance), and SDSW together with SWM, are considered as indexes for steering wheel management (Ariansyah et al., 2018). Performance measure from three pilot-tests are compared in Table 2, with their corresponding rank. Considering SDLP, Driver 1 was the best performed driver in lane-keeping performance among all the three participants, then was the Driver 2 and Driver 3; while considering the SDSW and SWM, Driver 2 got a better steering wheel management than Driver 3 and Driver 1.

Table 2. Comparison of performance measures

\begin{tabular}{|c|c|c|c|}
\hline Performance Index & $\begin{array}{c}\text { Driver } \\
1\end{array}$ & $\begin{array}{c}\text { Driver } \\
2\end{array}$ & Driver 3 \\
\hline SDLP & $2.0756(1 \mathrm{st})$ & $2.1144(2 \mathrm{nd})$ & $2.4519(3 \mathrm{rd})$ \\
\hline LPM & $3.4946(2 \mathrm{nd})$ & $3.1685(1 \mathrm{st})$ & $4.3033(3 \mathrm{rd})$ \\
\hline SDSW & $0.1594(3 \mathrm{rd})$ & $0.1476(1 \mathrm{st})$ & $0.1492(2 \mathrm{nd})$ \\
\hline SWM & $0.066(3 \mathrm{rd})$ & $0.0551(1 \mathrm{st})$ & $0.0606(2 \mathrm{nd})$ \\
\hline
\end{tabular}

User data of High Frequency component of heart rate variability (HRV-HF) is recorded and normalized as shown in Figure 5, being arranged by time series of sub-tasks in the X-axis. The sampling time frame for each sub-task was 10s x 3 times, positioning at the middle of each task phase. In the Adaptation session, the value of normalized HRV-HF in the first manual control driving phase was 0.745 ( $\mathrm{SD}=0.379)$, in the autonomous driving phase was $0.839(\mathrm{SD}=0.249)$, and in the second manual control driving phase (post Take-over) was 0.903 ( $\mathrm{SD}=0.304$ ). In the Test session, the value of normalized HRV-HF in the first manual control driving phase was $0.806(\mathrm{SD}=0.660)$, in the autonomous driving phase was $0.610(\mathrm{SD}=0.300)$, and in the second manual control driving phase (post Take-over) was $0.749(\mathrm{SD}=0.330)$.

As one of the indexes for the evaluation of the cognitive workload, the change of HRV-HF in the Adaptation session and Test session suggests that user cognitive workload increased in the Adaptation session with time, including $\mathrm{AD}$ phase that the user did not need to operate, and decreased in the Test session with time generally $(\mathrm{P}=0.17>0.05 ; \mathrm{P} 1=0.46, \mathrm{P} 2=0.08, \mathrm{P} 3=0.07)$, reached its lowest value in $\mathrm{AD}$ phase particularly. In general, from the cognitive workload evaluated by HRV-HF, user engaged more in the Adaptation session than in the Test session. It may be affected by the novelty of trying the $\mathrm{AD}$ function for the first time in the Adaptation session, therefore when user repeated the operation in the Test session, their cognitive workload decreased.

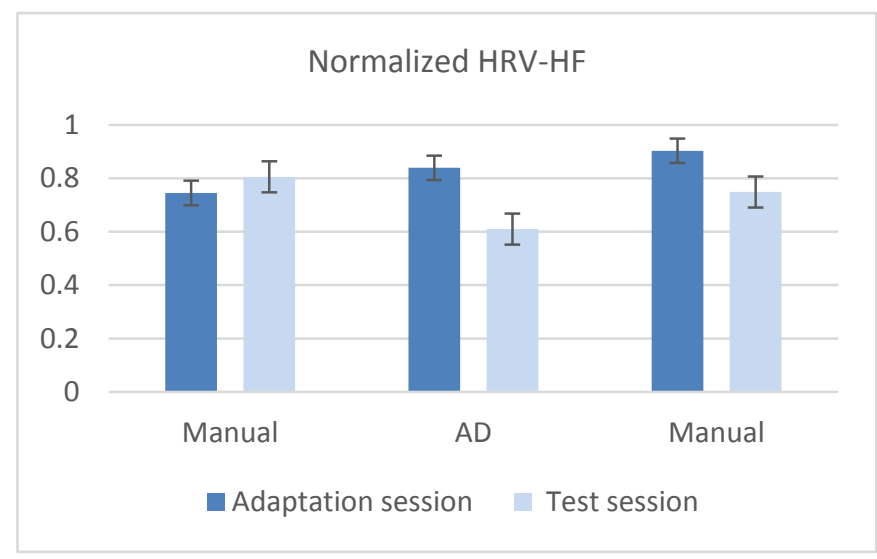

Figure 5. Comparison of Normalized HRV-HF in Adaptation session and Test session

The variation in skin conductance demonstrated how the user reacted to the driving environment and stimuli in Adaptation session and Test session as shown in Figure 6. Specifically, in Adaptation session, the value of normalized skin conductance in the first manual control driving phase was 0.055 $(\mathrm{SD}=0.039)$, in the $\mathrm{AD}$ phase was 0.021 ( $\mathrm{SD}=0.006)$, and in the post-Take-over manual control driving phase was $0.054(\mathrm{SD}=0.047)$; in Test session, the value of normalized skin conductance in the first manual control driving phase was 0.032 ( $\mathrm{SD}=0.016$ ), in the $\mathrm{AD}$ phase was $0.018(\mathrm{SD}=0.005)$, and in the post-Take-over manual control driving phase was $0.033(\mathrm{SD}=0.011)$. 
The result of skin conductance measurement suggests that in general user had lower cognitive arousal in the Test session respect to the Adaptation session $(\mathrm{P}=0.06>0.05)$, might be caused by the adaptation to the operation, which implies that an adaptation or tutorial session would be useful for smoothing user cognitive reaction in the semi-autonomous driving context. Particularly in the AD phase of both cases user gained lowest cognitive arousal, which means that they perceived less stimuli from the driving environment when they were not driving comparing to the manual control driving phases.

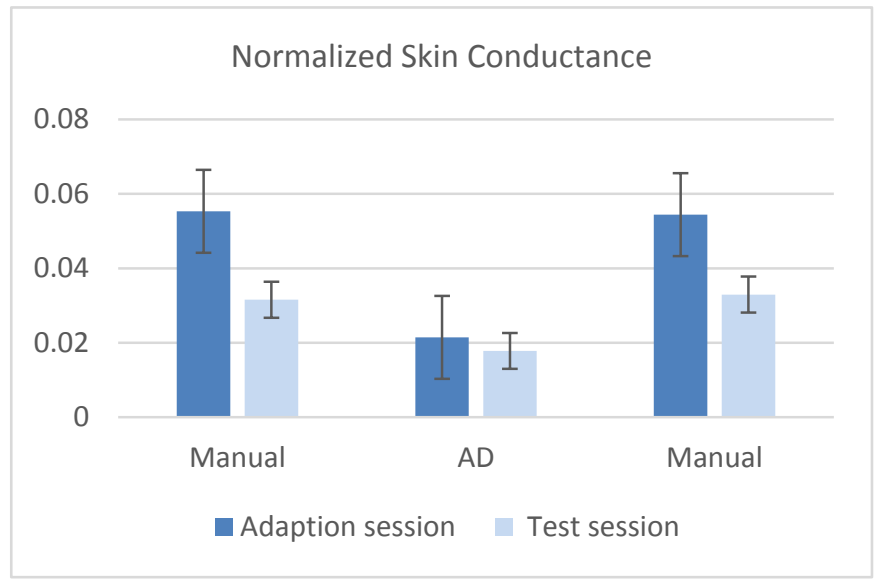

Figure 6. Comparison of Normalized Skin Conductance in Adaptation session and Test session

Take-over reaction time in this study was defined as the time from when the ADS requested the user to take-over control and regain the DDT, to when the user took action to operate the vehicle, i.e. turning the steering wheel, stepping on the throttle pedal or brake pedal. User's reaction time is shown in Table 3, from which we can see that Driver 1 was the most rapid driver among all these three participants, then was the Driver 3, and the last one is Driver 2 who used 7.2s to respond the request of Take-over. It might due to two reasons: the first one is that the Driver 2 probably had a high confidence of his driving technique and he believed that he can resume the control even not respond to the system in time. Because according to the pre-test questionnaire, he ranked his own driving skill 9 out of 10 (Driver 1 ranked 8, Driver 3 ranked 6), which was the highest in three participants. The second reason could be that the state of the driver at that moment was out-of-loop, neither in physical control nor monitoring the driving environment, thus he was not able to react in time for any kind of request from the ADS.

Table 3. User reaction time to Take-over request

\begin{tabular}{|c|c|c|c|}
\hline & Driver 1 & $\begin{array}{c}\text { Driver } \\
2\end{array}$ & Driver 3 \\
\hline Reaction Time $[s]$ & 1.1 & 7.2 & 2.1 \\
\hline
\end{tabular}

Evaluating three driver's performance in the semi-autonomous driving context has been done with the driving simulator. Driver's performance was evaluated from various aspects: behavioural measurements assessing the ability of speed control (Time-distance comparison), lane keeping performance (SDLP), steering wheel management (SDSW together with SWM), and Reaction time to Take-over request; and physiological measurement assessing the cognitive workload of user in different phase of driving tasks (HRV-HF), and Cognitive arousal level to the driving environment (Skin Conductance).

The Swedish Occupational Fatigue-20 Inventory (SOFI-20) was administered to the participants after each session. Five subjective dimensions of the SOFI questionnaire includes: discomfort, lack of energy, lack of motivation, physical exertion, and sleepiness. Three drivers did not show obvious disagreement on the facts that this experiment was low graded in Lack of energy, Physical exertion, Physical discomfort, and Lack of motivation aspects, but Driver 2 expressed a different evaluation on the Sleepiness aspect, with a 10-times higher sum-score than the media sum-score of other two drivers. It might be a support for the assuming that before Take-over he was out-of-the-loop.

From this experiment, it is shown that the user adapted to the ADS as a novel function in a relative short time, because their cognitive load decreased obviously in the Test session comparing to the 
Adaptation session $(\mathrm{P}=0.17>0.05)$, and during the $\mathrm{AD}$ phase, user showed lower perception to the driving circumstances $(\mathrm{P}=0.06>0.05)$.

Considering the drivers' performance from the measurement of speed control, lane-keeping and steering wheel management, Driver 2 performed better than the others, then there was Driver 1 and Driver 3. Hence, $\mathrm{H} 1$ is supported by the results. In order to verify the H2, a matrix ranking was created by taking the four indexes of driver performance (Speed control, Lane-keeping, Steering wheel management, and Reaction time to Take-over request). To check the correlation of these four indexes, the Kendall's coefficient of concordance was introduced, and the result $(\mathrm{W}=0.188<0.3)$ showed that these indexes had very low consistency in evaluating the drivers' performance. It implied that there did not exist a high correlation between the take-over response time and the user performance to support $\mathrm{H} 2$, which means that we cannot anticipate a driver's reaction time to the take-over request by analysing his past driving performance.

\section{CONCLUSION}

The present study proposed a method to explore human factors in the semi-autonomous driving context, using driving simulator. The transition of control in different phases for a semi-autonomous vehicle was fully considered in the study. Moreover, with user behavioural data acquisition integrated, a wide scope of indexes become available in the user performance evaluation: standard deviation of lateral position (SDLP), standard deviation of steering wheel angle (SDSW), mean amplitude of steering wheel (SWM), and physiological signals such as Heart rate variability and Electrodermal activity. A pilot experimental test of three participants was conducted using this protocol. The results confirmed the feasibility of the method to categorize user performance by behavioural data from the driving simulator, however, were not statistically significant due to the limited sample size. Specifically, it was revealed in the pilot test that there was no significant positive correlation between a driver's driving skill and his reaction time to the Takeover request, which would be verified in the future research with a larger range of participant. Further studies are needed with a longer $\mathrm{AD}$ phase, to focus on detecting and evaluating the driver's state, and to distinguish if the driver is on the loop or out of the loop and to find the corresponding solutions.

\section{ACKNOWLEDGEMENTS}

This research was supported by the i.Drive Lab (Interaction of Driver, Road, Infrastructure, Vehicle, and Environment - Politecnico di Milano - http://www.idrive.polimi.it/). The authors are thankful to participants for investing their time in this study.

\section{REFERENCES}

Åhsberg, E., Garnberale, F. and Kjellberg, A. (1997), "Perceived quality of fatigue during different occupational tasks development of a questionnaire", International Journal of Industrial Ergonomics, Vol. 20 No. 2, pp. 121-135. https://doi.org/10.1016/S0169-8141(96)00044-3.

Ariansyah, D., Caruso, G., Ruscio, D. and Bordegoni, M. (2018), “Analysis of autonomic indexes on driver's workload to assess the effect of visual ADAS on user experience and driving performance in different driving conditions", Journal of Computing and Information Science in Engineering, Vol. 18 No. 3, http://dx.doi.org/10.1115/1.4039313.

Banks, V.A. and Stanton, N.A. (2017), "Analysis of driver roles: Modelling the changing role of the driver in automated driving systems using EAST", Theoretical Issues in Ergonomics Science, https://doi.org/10.1080/1464922X.2017.1395465.

Banks, V.A., Eriksson, A., O'Donoghue, J. and Stanton, N.A. (2018), "Is partially automated driving a bad idea? Observations from an on-road study", Applied ergonomics, Vol. 68, pp. 138-145. https://doi.org/10.1016/j.apergo.2017.11.010.

Blanco, M., Atwood, J., Vasquez, H.M., Trimble, T.E., Fitchett, V.L., Radlbeck, J., and Morgan, ... and F, J. (2015), "Human Factors Evaluation of Level 2 and Level 3 Automated Driving Concepts", Proceedings of the Transportation Research Board Annual Meeting, Vol. 93, https://doi.org/10.13140/RG.2.1.1874.7361, 2014.07, 320.

Brookhuis, K.A. and de Waard, D. (2010), “Monitoring drivers' mental workloadin driving simulators using physiological measures", Accident Analysis and Prevention, Vol. 42 No. 3, pp. 898-903. https://doi.org/10.1016/j.aap.2009.06.001.

Reimer, B. and Mehler, B. (2011), "The impact of cognitive workload onphysiological arousal in young adult drivers: a field study and simulation validation”, Ergonomics, Vol. 54 No. 10, pp. 932-942.

https://doi.org/10.1080/00140139.2011.604431. 
Casner, S.M., Hutchins, E.L. and Norman, D. (2016), “The challenges of partially automated driving”, Communications of the ACM, Vol. 59 No. 5, pp. 70-77. https://doi.org/10.1145/2830565.

Drosdol, J. and Panik, F. (1985), "The Daimler-Benz Driving Simulator A Tool for Vehicle Development”, $S A E$ Transactions, Vol. 94, pp. 981-997. http://www.jstor.org/stable/44467637.

De Winter, J., Van Leuween, P. and Happee, P. (2012), “Advantages and disadvantages of driving simulators: A discussion”, In: A. J. Spink, F. Grieco, O. E. Krips, L. W. S. Loijens, L. P. J. J. Noldus and P. H. Zimmermann, (Ed.), Proceedings of the 8th international conference on methods and techniques in behavioral research, Noldus Information Technology bv, Utrecht, The Netherlands, pp. 47-50.

Eriksson, A. and Stanton, N.A. (2017), "Driving performance after self-regulated control transitions in highly automated vehicles”, Human factors, Vol. 59 No. 8, pp. 1233-1248. https://doi.org/10.1177/0018720817728774.

Gertman, D.I. and Blackman, H.S. (1994), Human reliability and safety analysis data handbook, John Wiley and Sons.

Heikoop, D.D., de Winter, J.C., van Arem, B. and Stanton, N.A. (2018), “Acclimatizing to automation: driver workload and stress during partially utomated car following in real traffic". Manuscript submitted.

Louw, T. and Merat, N. (2017), "Are you in the loop? Using gaze dispersion to understand driver visual attention during vehicle automation”, Transportation Research Part C: Emerging Technologies, Vol. 76, pp. 35-50. https://doi.org/10.1016/j.trc.2017.01.001.

Logitech. G920 driving force racing wheel and pedals. https://www.logitechg.com/enroeu/products/driving/driving-force-racing-wheel.html.

Merat, N., Jamson, A.H., Lai, F.C., Daly, M. and Carsten, O.M. (2014), "Transition to manual: Driver behaviour when resuming control from a highly automated vehicle", Transportation research part F: traffic psychology and behaviour, Vol. 27, pp. 274-282. https://doi.org/10.1016/j.trf.2014.09.005.

Merat, N., Seppelt, B., Louw, T., et al. (2018), "The “out-of-the-loop" concept in automated driving: Proposed definition, measures and implications", Cognition, Technology and Work, pp. 1-12. https://doi.org/10.1007/s10111-018-0525-8.

Mercedes-Benz (2013), Distronic Plus with Steering Assist. Available at https://www.mercedes-benz.com. [Accessed 10.12.2018].

Naujoks, F., Purucker, C. and Neukum, A. (2016), "Secondary task engagement and vehicle automationComparing the effects of different automation levels in an on-road experiment", Transportation research part F: traffic psychology and behaviour, Vol. 38, pp. 67-82. https://doi.org/10.1016/j.trf.2016.01.011.

Ruscio, D., Bascetta, L., Gabrielli, A., et al. (2017), "Collection and comparison of driver/passenger physiologic and behavioural data in simulation and on-road driving", Models and Technologies for Intelligent Transportation Systems (MT-ITS), 2017 5th IEEE International Conference on, IEEE, pp. 403-408. https://doi.org/10.1109/MTITS.2017.8005705.

SAE J3016 (2016), Taxonomy and Definitions for Terms Related to Driving Automation Systems for On-Road Motor Vehicles, J3016-201609: SAE International.

SAE J3114 (2016), Human Factors Definitions for Automated Driving and Related Research Topics, J3114201612: SAE International.

Saffarian, M., de Winter, J.C. and Happee, R. (2012, September), “Automated driving: human-factors issues and design solutions", Proceedings of the human factors and ergonomics society annual meeting, Vol. 56 No. 1, pp. 2296-2300. Sage, Los Angeles. https://doi.org/10.1177/1071181312561483.

Shen, S. and Neyens, D.M. (2017), “Assessing drivers' response during automated driver support system failures with non-driving tasks", Journal of safety research, Vol. 61, pp. 149-155. https://doi.org/10.1016/j.jsr.2017.02.009.

Strand, N., Nilsson, J., Karlsson, I.M. and Nilsson, L. (2014), "Semi-automated versus highly automated driving in critical situations caused by automation failures", Transportation Research Part F: Traffic Psychology and Behaviour, Vol. 27, pp. 218-228. https://doi.org/10.1016/j.trf.2014.04.005.

Strayer, D.L. and Drew, F.A. (2004), "Profiles in driver distraction: Effects of cell phone conversations on younger and older drivers", Human factors, Vol. 46 No. 4, pp. 640-649. https://doi.org/10.1518/hfes.46.4.640.56806.

Taheri, S.M., Matsushita, K. and Sasaki, M. (2017), "Development of a Driving Simulator with Analyzing Driver's Characteristics Based on a Virtual Reality Head Mounted Display”, Journal of Transportation Technologies, Vol. 7 No. 03, pp. 351. http://dx.doi.org/10.4236/jtts.2017.73023.

Tesla Motors (2016), Model S Software Version 7.0, https://www.tesla.com/en_GB/presskit/autopilot.

Thought technology. . Physiological sensors, http://thoughttechnology.com/.

Unity 3D. https://unity.com/.

Yao, L., Liu, Y., Li, W., Zhou, L., Ge, Y., Chai, J. and Sun, X. (2014, June), "Using physiological measures to evaluate user experience of mobile applications", International Conference on Engineering Psychology and Cognitive Ergonomics, Cham, Springer, pp. 301-310. 